\title{
O Papel Transformador do Estudante de Medicina no Cenário da Endemia de Hanseníase no Brasil: Relato de Experiência
}

\section{The Transformative Role of the Medical Student in relation to the Leprosy Endemic in Brazil: Case Report}

\section{PALAVRAS-CHAVE \\ - Educação Médica; \\ - Internato e Residência; \\ - Atenção Primária à Saúde; \\ - Hanseníase.}

\section{KEYWORDS}

- Medical Education;

- Internship and Residency;

- Primary Health Care;

- Leprosy.

Recebido em: 21/11/2014

Reencaminhado em: 08/09/2015

Aprovado em: 05/04/2016
Milena Marchini Rodrigues ${ }^{I}$ Ianê Cardoso Diniz Ana Rita Barbieri ${ }^{I}$

Luiza Helena de Oliveira Cazola ${ }$ Joaquim Dias Mota Longo ${ }^{I}$ Alexandre Moretti de Lima ${ }^{I}$

\section{RESUMO}

Durante o Internato em Saúde da Comunidade, os acadêmicos de Medicina vivenciaram uma experiência em que foi possível desenvolver habilidades e atitudes na resolução de um caso de diagnóstico tardio de hanseniase. Trata-se de um estudo descritivo de abordagem qualitativa, envolvendo uma Unidade Básica de Saúde da Família em Campo Grande (MS) e a Faculdade de Medicina da Universidade Federal de Mato Grosso do Sul. O acompanhamento desta paciente permitiu reflexões e aprendizado quanto à necessidade de realizar exame físico e, com base em protocolos para diagnóstico e tratamento da hanseníase, assegurar o acompanhamento e seguimento de um caso que permaneceu por mais de dois anos sem tratamento apesar dos evidentes sinais clínicos da doença. Por outro lado, o analfabetismo, a ocupação e o espaço geográfico da paciente contribuíram para o agravamento do quadro. Este estudo de caso favoreceu uma visão ampliada da formação de profissionais, que praticaram a integração dos diferentes campos de conhecimento para garantir a assistência integral à paciente.

\section{ABSTRACT}

Medical students during their Internship in Community Health hadan experience in which it was possible to develop skills and attitudes in solving a case of late diagnosis of leprosy. Thisis a descriptive qualitative study, involving a Basic Family Health Unit inCampo Grande (MS)and the Federal University of Mato Grosso do SulMedicine School. Following the patient's case allowed for reflections and learning regarding the need to perform physical examination and, based on procedures for leprosy diagnosis and treatment, to ensure the adequate follow-up and monitoring of a case in which the patient was not treated for more than two years, despite the obvious symptoms of the disease. Moreover, the patient's illiteracy, occupation and geography contributed to condition worsening. This case study favored an expanded view on the training of professionals, who practiced the integration of different fields of knowledge to ensure comprehensive patient care. 


\section{INTRODUÇÃO}

A formação contemporânea do profissional médico deve ser consolidada no tripé ensino, pesquisa e extensão. Esta formação proporciona ao estudante condições para adquirir conhecimentos, habilidades e atitudes que o tornem competente para o exercício profissional, com postura crítica e proativa frente aos problemas de saúde da população ${ }^{1}$.

Segundo as Diretrizes Curriculares Nacionais do Curso de Graduação em Medicina, definidas pelo Ministério de Educação e Cultura em 2014, a formação profissional do médico deve ser generalista, humanista, crítica e ética, e deve capacitá-lo a atuar no processo de saúde e doença em seus diferentes níveis de atenção².

Com o foco no ensino baseado no desenvolvimento de competências, a Faculdade de Medicina da Universidade Federal de Mato Grosso do Sul (Famed/UFMS) oferece aos acadêmicos do quinto ano o Internato em Saúde da Comunidade, com duração de nove semanas, cujo objetivo é vivenciar a prática médica de uma Unidade Básica de Estratégia de Saúde da Família (UBSF).

Nesse período, os estudantes desenvolvem um diagnóstico situacional de saúde do território da sua equipe, analisam e interferem na melhoria de alguns indicadores locais definidos a partir do Pacto pela Saúde, acompanham estudos de casos e realizam atendimentos clínicos com uso de pontos de referências hospitalares e ambulatoriais.

As atividades são acompanhadas por um médico preceptor da própria rede municipal de saúde de Campo Grande (MS) e por docentes da UFMS, o que favorece a troca de saberes, a qualidade na formação e a transformação da realidade assistencial.

Este relato descreve um caso vivenciado por acadêmicos durante o Internato em Saúde da Comunidade, identificado em meio à demanda espontânea dos atendimentos médicos ambulatoriais como um possível caso de hanseníase.

O Brasil ainda detém o maior número de casos de hanseníase nas Américas e o segundo maior do mundo, atrás apenas da Índia ${ }^{3,4}$. Nos últimos 20 anos, dos 122 países considerados endêmicos em 1985, 107 conseguiram erradicar a doença com o advento da poliquimioterapia, adotada como tratamento preferencial no mundo todo. Em 2011, foram registrados 33.955 casos no País, com prevalência de 1,54/10.000 habitantes. O Centro-Oeste tem a segunda mais alta taxa de prevalência: 3,3 casos/10.000 habitantes em $2005^{3}$.

Como medida para diminuir essas taxas, o Ministério da Saúde propôs a implantação do tratamento poliquimioterápico a partir do diagnóstico clínico. O diagnóstico e o tratamento precoce previnem incapacidades e cessam a transmissão. $\mathrm{Na}$ maioria dos casos, uma anamense completa e um bom exame dermatoneurológico identificam a doença, sendo suficientes para iniciar o tratamento, sempre com atenção, pois a hanseníase possui evolução variável e espectral e é preciso considerar também diagnósticos diferenciais de comorbidades ${ }^{5}$.

O ensino do diagnóstico da hanseníase é um importante aspecto da formação médica no campo da saúde pública no Brasil. Além da necessidade da investigação clínica para diagnóstico e resolução de casos, a dimensão social da saúde e o contexto econômico e cultural em que vive a maior parcela da população brasileira precisam ser considerados na abordagem ao paciente. Propiciar ao aluno de Medicina a vivência dessas circunstâncias das quais emergem as condições de saúde e seus agravos - é fundamental na formação das competências do médico generalista, um profissional comprometido com a integralidade do atendimento ao usuário e atento às necessidades da área onde atua.

\section{IDENTIFICAÇÃO DO PROBLEMA E ATUAÇÃO DA EQUIPE}

Com a participação da médica preceptora na escolha do estudo de caso, decidiu-se investigar o quadro dermatológico sugestivo de hanseníase sem diagnóstico definitivo e que havia perdido seguimento na UBSF.

Diante da perda do seguimento, o primeiro passo foi realizar a busca da paciente. Por meio de uma foto que a médica possuía para documentar as lesões, uma agente comunitária de saúde (ACS) a identificou e conduziu as acadêmicas à visita domiciliar, que ocorreu em abril de 2014. A paciente foi solícita, colaborativa e mostrou-se feliz e aliviada com a possibilidade de diagnóstico para sua doença.

\section{HISTÓRIA DA PACIENTE E INTERVENÇÕES}

A paciente é do sexo feminino, 50 anos, analfabeta, dona de casa, natural de Corumbá (MS) e viveu em área rural durante 35 anos. Mudou-se para Campo Grande em 2012, tendo procurado a UBSF em novembro com queixa de nódulos disseminados em membros superiores havia um ano.

O primeiro contato da paciente com a UBSF foi em em janeiro de 2013, quando apresentava pápulas e placas eritematosas pruriginosas em membros inferiores havia um ano. Retornou em março de 2013 com exames laboratoriais bioquímicos e reumatológicos normais, tendo sido receitados sintomáticos para prurido. Em novembro do mesmo ano, retornou à UBSF com a mesma queixa. Realizada a hipótese diagnóstica de hanseníase, foi solicitado Baar na linfa, hemograma e bioquímica.

Sem diagnóstico e com os resultados de exames laboratoriais, que segundo o relato dos profissionais de saúde não apresentavam alterações, a paciente foi encaminhada para serviço de referência em Dermatologia e desde então não realizou mais acompanhamento na UBSF. A consulta com o derma- 
tologista ocorreu em dezembro de 2013, tendo sido solicitada biópsia da lesão, que, por falta de compreeensão da paciente, não foi realizada.

Em abril de 2014, no primeiro contato dos acadêmicos, em visita domiciliar, a paciente apresentava nódulos disseminados em face, tronco, abdome e membros superiores e alguns em membros inferiores; infiltração lobular direita; conjuntivas oculares hiperemiadas bilateralmente. Referiu ardência nas lesões, negou prurido, cãibras e formigamentos. Apresentava teste da sensibilidade térmica e tátil alterado em todo o corpo; sensibilidade corneana preservada; nervos sem espessamento ou dor a palpação.

A história da paciente e os evidentes sinais clínicos, após o exame dermatoneurológico, confirmaram o diagnóstico de hanseníase virchowiana. Foram solicitados hemograma e bioquímica para exclusão de outras comorbidades e controle inicial das funções hematopoéticas, hepáticas e renais para iniciar o tratamento com a poliquimioterapia (PQT).

Ao buscarem os resultados dos exames no sistema informatizado da UBSF, as acadêmicas encontraram o resultado do exame do Baar na linfa solicitado em novembro de 2012; diferentemente das informações fornecidas pelos profissionais de saúde, o resultado era positivo: 5+/6+. Imediatamente, a PQT multibacilar foi instituída, e o caso notificado pela equipe da saúde da família.

Em consulta no Hospital Universitário, foi realizada a biópsia da paciente, para documentação histopatológica. $\mathrm{O}$ fragmento de pele apresentava, na derme, infiltração difusa constituída por histiócitos vacuolados contendo globias de Baar íntegros. Tal infiltrado foi separado da epiderme por faixa de Unna de tecido conjuntivo, sendo conclusivo para hanseníase virchowiana ${ }^{6}$.

Figura 1

Linha do cuidado da paciente conforme registros do prontuário. Campo Grande, 2014.

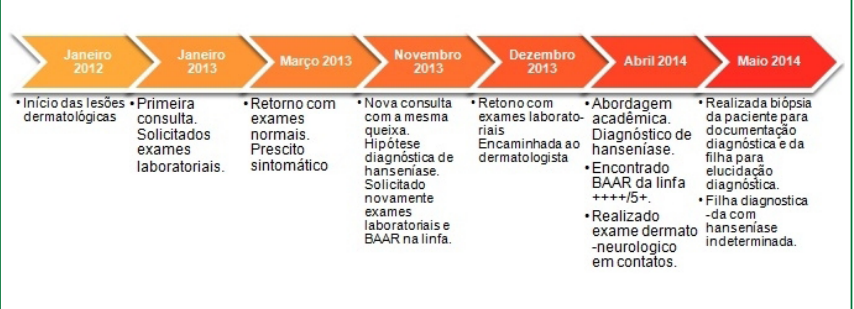

Em razão da baixa escolaridade e dificuldades em assimilar as informações, a paciente não compreendeu como seria o tratamento e então foi instituída a tomada diária do medicamento na Unidade de Saúde. Após dois meses de tratamento, no encerramento do período do internato, apresentava melhora significativa das lesões (Figura 2) e gratidão visivelmente expressa em suas atitudes, observadas diariamente na UBSF.

\section{Figura 2}

Evolução das lesões cutâneas da paciente sem tratamento e após 45 dias de tratamento. Campo Grande, 2014.

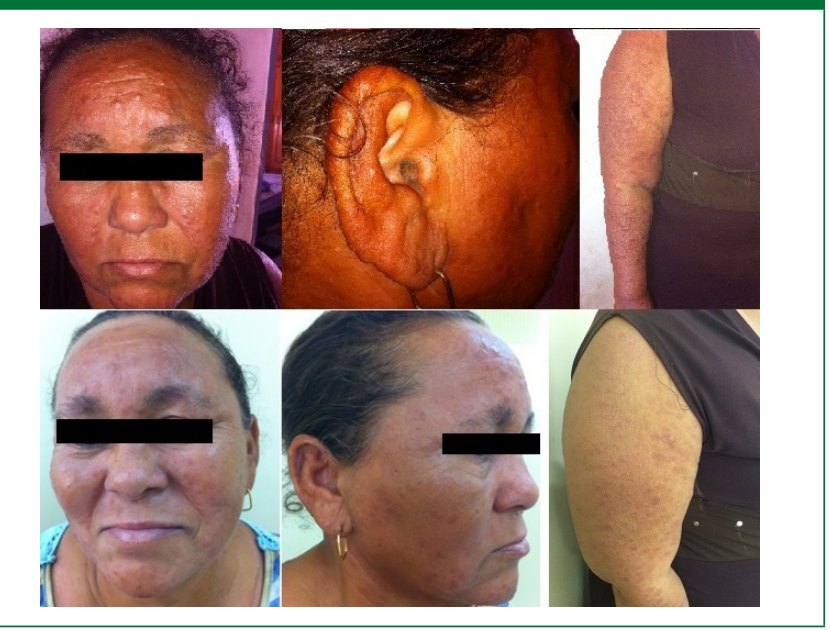

\section{INVESTIGAÇÃO DOS CONTATOS INTRADOMICILIARES}

Para realização do exame dermatoneurológico dos comunicantes intradomiciliares (CI) da paciente, os familiares foram conduzidos ao hospital universitário, dada a dificuldade de fechar o diagnóstico de seu neto de três anos. Na consulta com o dermatologista, foram examinados: genro (30 anos), filha (23 anos) e neto ( 3 anos). $\mathrm{O}$ genro não apresentou alterações no exame dermatoneurológico.

O neto apresentava madarose, que a mãe referiu ser constitucional, e mácula hipocrômica em dorso. Porém, pela pouca idade, não houve colaboração para realização do teste de sensibilidade térmica e tátil. Os testes da histamina e da pilocarpina foram negativos e optou-se por não iniciar o tratamento e agendar seu retorno em seis meses.

A filha apresentava madarose bilateral e mácula hipocrômica com alteração da sensibilidade térmica e tátil em dorso e realizou teste da pilocarpina, que resultou alterado tanto em área de mácula como em área aparentemente sadia. Resultado negativo para Baar na linfa. Foi realizada biópsia da lesão com diagnóstico histológico de hanseníase indeterminada. $\mathrm{O}$ tratamento foi iniciado para hanseníase paucibacilar (PB). 


\section{ANÁLISE DA EXPERIÊNCIA}

A endemia hansênica está no limiar da sua eliminação como problema global de saúde pública. O Brasil, porém, é o único país da América Latina onde a doença ainda não foi erradicada, classificando-se em segundo lugar em prevalência no mundo $^{7}$. Desde a década de 1990, a assistência foi difundida em todos os níveis de atenção e serviços de saúde, e desde os anos 2000 o Ministério da Saúde preconiza que a atenção básica seja o principal ponto de atenção para o diagnóstico, tratamento e acompanhamento dos pacientes acometidos ${ }^{8,9}$. Diversos estudos apontam que a descentralização melhorou a detecção de casos da doença, com aumento no registro dos mesmos ${ }^{10}$.

A distribuição da hanseníase na população brasileira é profundamente desigual no que diz respeito aos espaços geográficos e às condições econômicas e sociais, como ocupação, escolaridade e renda ${ }^{11,12,13}$. Alguns estudos apontam que, em geral, os habitantes de áreas rurais não só têm dificuldade em acessar os serviços de saúde, como também recebem diagnósticos tardios ${ }^{14,15}$. O ensino médico deve ofertar conteúdos e práticas que promovam a construção de competências profissionais que superem a tríade agente-hospedeiro-ambiente e levem em consideração a complexidade da determinação social das doenças tanto nas populações quanto nos indivíduos ${ }^{16,17}$.

No âmbito do atendimento ao indivíduo, a relação a ser estabelecida necessita incorporar boas práticas clínicas. Além da queixa referida do paciente, cabe ao acadêmico em regime de internato médico dispor de um conjunto de conhecimentos para realizar indagações adicionais e exame físico minucioso e, assim, agregar à história clínica evidências de hipóteses diagnósticas que contemplem a integralidade do paciente em atendimento $^{17,18,19}$. Ele deve ser capaz de atuar com tecnologias de alta complexidade e de baixa densidade e compreender o contexto biopsicossocial da comunidade onde atua. Um estudo acerca da integralidade na formação médica apontou em seus resultados a necessidade de privilegiar na graduação uma visão integral sobre o usuário, compreendendo sua história de vida e contexto social ${ }^{20}$.

Em nossa experiência, a doença na forma multibacilar e a negligência em sua vigilância permitiram que a paciente continuasse como uma fonte de transmissão, gerando um caso intradomiciliar no período ${ }^{21}$. $\mathrm{O}$ resultado de uma pesquisa sobre o processo de trabalho em hanseníase apontou que a organização dos serviços nos municípios está estruturada no modelo clínico, e as práticas destinadas à abordagem coletiva são bastante pontuais ${ }^{21}$.

Os serviços de saúde, enquanto cenários para o ensino médico, se compõem desses limites e precisam de maior permeabilidade com o ensino, que tem privilegiado abordagens integrais ${ }^{22}$. $\mathrm{O}$ acadêmico de Medicina inserido nos serviços de atenção primária deve ser agente transformador dessa realidade, colocando em prática o ensino aprendido com as Novas Diretrizes Curriculares, como neste relato. O serviço de atenção primária ganha ao melhorar o nível de atenção em saúde e fortalecer os princípios do SUS, e os estudantes consolidam seu aprendizado em contato direto com a comunidade, fundamental para a formação dos futuros médicos ${ }^{22}$.

Para o combate à hanseníase, é necessária a vigilância dos pacientes diagnosticados no decorrer do tratamento e após a alta, para avaliar o grau de incapacidades, acompanhar recidivas e manejar eventuais surtos reacionais ${ }^{23}$. Da mesma forma, é preciso instituir redes de comunicação entre municípios para que comunicantes sejam avaliados. Tais ações são necessárias para reduzir os danos e investigar a cadeia de transmissão da doença, considerando que seu período de incubação é, em média, de dois a cinco anos, com variações. A paciente, originária de área rural com precária assistência e saúde e com difícil acesso a outro município, pode ser mais uma pertencente a uma comunidade com significativa prevalência da doença.

No caso da paciente que gerou este relato, a ausência de um exame físicodermatoneurológico completo, lapsos no atendimento clínico e desconhecimento dos protocolos para diagnóstico e tratamento da hanseníase explicam o longo período (24 meses) vivido sem diagnóstico. Por outro lado, o analfabetismo, a ocupação e o espaço geográfico da paciente contribuíram para o agravamento do quadro e evidenciam a fragmentação dos processos de trabalho com enfoque predominantemente clínico.

Um relato de experiência de acadêmicos de Enfermagem envolvendo pessoas com sequelas de hanseníase numa instituição de Goiás demonstrou que 91,6\% dos pacientes estudados se enquadram em um dos maiores graus de incapacidade. Tal fato demonstra a dificuldade em realizar o tratamento precoce, permitindo franca instalação do bacilo e acometimento dermatoneurológico irreversível, com desenvolvimento da doença em sua forma mais debilitante. Isso remete à situação em que se encontrava a paciente referida neste relato, que já apresentava sequelas neurológicas e dermatológicas ${ }^{24}$.

Vivenciamos um período de transformação no ensino médico, no qual é fundamental consolidar um modelo de atenção que cuide de pessoas, e não de doenças. Uma das responsabilidades da equipe e do médico, em particular, é lidar com a concepção da atenção integral, indo além do mínimo necessário ${ }^{25}$. Há diversas ações voltadas para essa formação mais ampliada, como as novas Diretrizes Curriculares, práticas de educação permanente e incentivo a novas experiências, como o PET-Saúde e Pró-Saúde ${ }^{25}$. As diretrizes curriculares dos cursos 
de Medicina propiciaram uma nova relação ensino-aprendizagem com a participação dinâmica de todos os atores envolvidos e a percepção de sua realidade social na determinação da saúde das pessoas em sua integralidade e no relacionamento com as instituições de saúde.

\section{CONSIDERAÇÕES FINAIS}

O objetivo de fazer do Internato em Saúde da Comunidade um espaço pedagógico para a incorporação de práticas pelos futuros médicos com base na realidade vivenciada numa Unidade de Saúde da Família permitiu total envolvimento dos acadêmicos na resolução do agravo da paciente e sua família, instituindo tratamento numa paciente bacilífera. Com o objetivo inicial de cumprir uma atividade curricular, os acadêmicos localizaram, diagnosticaram e trataram uma doença infectocontagiosa com mais de dois anos de evolução, que foi transmitida,nesse período, a um contato intradomiciliar.

Diante dessa demanda social e de um leque de circunstâncias socioculturais nas quais o médico deve atuar, torna-se imprescindível a busca incessante por uma vertente de educação mais integralizada e abrangente durante a formação acadêmica. As estudantes inseridas no âmbito da Atenção Primária à Saúde tiveram a oportunidade de realizar práticas clínicas preconizadas pelo SUS, como a valorização das queixas e da história clínica da paciente e atenção centrada na pessoa. Os exames laboratoriais foram solicitados com acompanhamento dos resultados. O resultado (fortemente positivo) do primeiro exame de Baar na linfa foi encontrado nos registros do prontuário eletrônico. Foi realizada uma consulta de toda a família no ambulatório de Dermatologia do Hospital Universitário, com a coleta de biópsia para laudo histopatológico, e foi iniciado o tratamento em mãe e filha, interrompendo a cadeia de transmissão.

Somente uma abordagem com assistência integral pode gerar ações transformadoras para os usuários acometidos pela hanseníase. A qualificação dos profissionais, a vigilância epidemiológica ativa, a integralidade do cuidado - sempre baseadas na equidade, individualizando e atuando de acordo com as limitações de cada usuário-e a integração entre o ensino e a atenção primária, além de aperfeiçoarem a formação médica, contribuem sobremaneira para a eliminação da hanseníase no Brasil.

$\mathrm{O}$ estudo de caso apresentado expressa vividamente os dilemas até então estudados somente na literatura. Foi uma oportunidade para a prática dos princípios éticos do SUS, consolidação do aprendizado teórico em atendimento integral e solidário, visão ampla da complexidade da determinação social e combate à hanseníase, demonstrando a força motriz que os acadêmicos podem gerar na atenção primária, na qualidade do sistema e na qualidade de vida dos pacientes. $\mathrm{O}$ aprendizado foi significativo, com importantes e duradouros ganhos de competências que certamente serão propagadas em casos semelhantes encontrados na prática dos futuros médicos.

\section{REFERÊNCIAS}

1. Albuquerque GS, Mendes RRS, Nascimento RCS. Internato de Medicina Social em uma Unidade de Saúde da Família. Rev Baiana de Saúde Pública. 2012:36(3);851-9.

2. Brasil. Ministério da Educação. Câmara de Educação Superior. Resolução no 3, de 20 de junho de 2014. Institui Diretrizes Curriculares Nacionais do Curso de Graduação em Medicina e dá outras providências. Diário Oficial da República Federativa do Brasil, Brasília, DF, 23 jun. 2014. [capturado em 20 out. 2014].

3. Brasil. Ministério da Saúde. Sistema de Informação de Agravos de Notificação (Sinan) e base populacional do IBGE. Brasília, DF, 2014.

4. Lastoria JC, Abreu MAMM. Hanseníase: Diagnóstico e tratamento. Diagn. tratamento. 2012;7(4):173-9.

5. Li TSF, Miranda LQ, Bringel DM, Daxbacher ELR. Importância clínica no diagnóstico da hanseníase. Rev Hospital Universitário Pedro Ernesto. 2011;10(1):29-35.

6. Ura S, BarretoJA. Papel da biópsia cutânea no diagnóstico de hanseníase. Hansenol. int. 2004;29(2):141-4

7. Martelli CMT, Stefani MMA, Penna GO, Andrade ALSS. Endemias e epidemias brasileiras, desafios e perspectivas de investigação científica: hanseníase. Rev. bras. de epidemio. 2002;5(3):273-85

8. Brasil. RIPSA. Indicadores e dados básicos. Indicadores de morbidade. Taxa de hanseníase [capturado 8 out 2014]. Disponível em: http://tabnet.datasus.gov.br/cgi/deftohtm.exe?idb2012/d0206.def.

9. Nardi SMT Vânia Del'Arco Paschoal VDA, Chiaravalloti-Neto F, Zanetta DMT. Deficiências após a alta medicamentosa da hanseníase: prevalência e distribuição espacial. Rev. Saúde Públ. 2012;46(6):969-77.

10. Cunha MD, Cavaliere FAM, Hércules FM, Duraes SMB, Oliveira MLW, Matos Haroldo J. Os indicadores da hanseníase e as estratégias de eliminação da doença, em município endêmico do Estado do Rio de Janeiro, Brasil. Cad. Saúde Pública. 2007; 23(5):1187-97.

11. Mencaroni DA. Análise especial da endemia hansênica no município de Fernandópolis - SP. 2003. Ribeirão Preto; 2003. Doutorado [Dissertação] - Escola de Enfermagem da Universidade São Paulo.

12. Magalhães MCC, Rojas LI. Spatial differentiation of leprosy in Brazil. Rev. Epidemio Serv Saúde. 2007;16(2):75-84 
13. Tibúrcio CS et al. Diagnóstico da distribuição geográfica da hanseníase no Brasil conforme região, clima e período. Encontro Internacional de Produção Científica Cesumar. Maringá, 2009. Disponível em: <http://www.unicesumar.edu.br/epcc2009/anais/camila_souza_tiburcio.pdf $>$. Acesso em: 15 de out.2014.

14. Smith K., Humphreys J, Wilson M. Addressing the health disadvantage of rural populations: how does epidemiological evidence inform rural health policies and research? Aust J Rural Health. 2008; 16(2):56-66.

15. Santinha G. Cuidados de saúde e território: um debate em torno de uma abordagem integrada. Saúde soc. 2013; 22(3)815-29

16. Fonseca AF. O território e o processo saúde-doença. Organizado por Angélica Ferreira e Ana Maria D'Andrea Corbo. Rio de Janeiro: EPSJV/Fiocruz; 2007

17. Puttini RF, Pereira Junior A, Oliveira LR. Modelos explicativos em saúde coletiva: abordagem biopsicossocial e auto-organização. Physis. 2010;20(3):753-67

18. Franco ALS, Bastos ACS, Alves VS. A relação médico-paciente no Programa Saúde da Família: um estudo em três municípios do Estado da Bahia, Brasil. Cad. Saúde Pública. 2005;21(1):245-55.

19. Brasil. Ministério da Saúde. Secretaria de Assistência à Saúde. Coordenação de Saúde da Comunidade. Saúde da Família: uma estratégia para a reorientação do modelo assistencial. Brasília. DF, 1997.

20. Gonze GG, Silva GA. A integralidade na formação dos profissionais de saúde: tecendo valores. Physis. 2011; 21(1):129-46.

21. Lanza FM, Lana FCF. O processo de trabalho em hanseníase: tecnologias e atuação da equipe de saúde da família. Texto Contexto Enferm. 2011; 20:238-46.

22. Souza MCA, Mendonça MA, Costa EMA, Gonçalves SJC; Teixeira JCD, Almeida-Júnior EHR, et al. O Universitário Transformador na comunidade: a experiência da USS. Rev. Bras. Educ. Med. 2014;38(2):269-274
23. Longo JDM. Contribuição ao conhecimento da transmissão da hanseníase em Campo Grande, Mato Grosso do Sul, 2006. Campo Grande; 2006. Doutorado [Dissertação] - Programa Multi-Institucional de Pós Graduação em Ciências da Saúde - Rede Centro Oeste da Universidade Federal de Mato Grosso do Sul.

24. Pereira SVM, Bachion MM, Souza AGC, Vieira SMS Avaliação da Hanseníase: relato de experiência de acadêmicos de enfermagem. Rev. Bras. Enferm. 2008; 61:774-80

25. Lopes RE, Malfitano APS, Palma AM, Furlan PG, Brito EM. Educação e saúde: territórios de responsabilidade, comunidade e demandas sociais. Rev. bras. Educ. Med. 2012;36(1):18-26.

26. Santos DL, Gerhardt TE. Desigualdades sociais e saúde no Brasil: produção científica no contexto do Sistema Único de Saúde. Rev Gaúcha Enf. 2008;29(1):29-36

\section{CONTRIBUIÇÃO DOS AUTORES}

Milena Marchini Rodrigues e Ianê Cardoso Diniz acompanharam e desenvolveram o caso clínico, organização do trabalho e descrição dos resultados. Ana Rita Barbieri, Luiza Cazola e Joaquim Dias da Motta Longo conceberam o desenho do estudo, discutiram os resultados e realizaram a revisão do manuscrito. Alexandre Moretti de Lima acompanhou o caso clínico e colaborou na organização do estudo e na discussão dos resultados.

\section{CONFLITO DE INTERESSES}

Os autores declaram não haver conflito de interesses.

\section{ENDEREÇO PARA CORRESPONDÊNCIA}

Joaquim Dias Mota Longo

Cidade Universitária, s/n - Unidade 9

Cidade Universitária - Campo Grande

CEP: 79070-900 MS

E-mail:jdmlongo@gmail.com 